\title{
Practicalities
}

(Vol. I. No. 5)

\section{THE CLINICAL STUDY OF THE CARDIO-VASCULAR SYSTEM}

\author{
By F. CROXON DELLER, M.D., D.A.
}

.PART III

\section{Hypertensive disease, the diagnosis of valvular murmurs and the significance of the} systolic murmur

The diagnosis of hypertension is dependent upon the finding of a heightened blood pressure by sphygmomanometric methods. It has already been emphasised, however, that the height of the diastolic pressure is of greater value in diagnosis than the systolic pressure. It was shown in the Case Report in Part I how excitement or emotion may cause a distinct rise in the systolic pressure, due to an increased cardiac output, without affecting the diastolic pressure. This type is known as systolic hypertension.

The diagnosis of diastolic hypertension would be considered if it was found that, after rest $\dot{N}$ the diastolic reading was over $90 \mathrm{Hg}$. The two most common causes of such increased + pressure are essential hypertension, and chronic nephritis. Should it be impossible to 0 implicate either of these pathological conditions, then one must remember that there are many other causes of diastolic hypertension. A. Rae Gilchrist ${ }^{1}$ proposed the following $\stackrel{\circ}{=}$ classification, which includes the two conditions already mentioned.

A. Neurological.

\section{Extra-Renal Origin}

(I) Emotional or psychic states.

(2) Increased intracranial pressure.

(3) Mid-brain and brain-stem lesions.

(a) poliomyelitis, (b) encephalitis, (c) trauma.

(4) Diencephalic syndrome.

B. Endocrinal.

(I) Suprarenal dysfunction.

(a) Paroxysmal hypertension (pheochromocytoma).

(b) adreno-genital syndrome (cortical adenoma).

(2) Pituitary adenoma.

(a) Cushing's basophilic syndrome.

(3) Ovarian hypofunction.

(a) menopausal.

(b) ? ovarian neoplasms.

(4) ? Placenta.

(a) Certain pregnancy toxaemias.

C. Peripheral (arteriolar spasm or sclerosis).

(I) Toxic origin: lead.

(2) UNKNOWN-“essential hypertension."

A Occlusive vascular disease.

\section{Renal Ischaemia}

(I) Renal arteriolar sclerosis.

(2) Stenosis of a main renal artery.

(3) Coarctation of the aorta.

(4) Amyloidosis.

(5) Infarction, thrombosis, trauma.

(6) Congestive heart failure.

(7) Aberrant renal artery.

(8) Periarteritis nodosa, disseminated lupus erythematosus. 
B. Primary Renal disease.

(I) Chronic glomerulo-nephritis.

(2) Chronic pyelonephritis.

(3) Renal tumours.

(4) Polycystic disease.

(5) Nephroptosis: postural hypertension.

C. Renal reflux (impedance to urinary outflow).

Hydronephrosis: congenital or acquired

(I) bilateral:

(a) urethral stricture.

(b) prostatic obstruction.

(c) vesical neoplasms.

(d) pelvic tumours, ? pregnant uterus.

(e) "spinal" bladder.

(2) unilateral:

(a) impacted calculus.

(b) ureteral strictures.

(c) sympathetic imbalance.

Only certain of these will be discussed here, but the other causes will have to be considered in the differential diagnosis of any case.

Essential hypertension is the most common cause of high blood pressure. There is no evidence, either clinical or pathological, that it is due to a primary inflammation of the kidneys, and such renal lesions as are found are almost certainly secondary in nature. It is a chronic condition, appearing in the later period of life, usually between the ages of 50 and 70 , and more often in the male sex. It is of rare appearance before 40 years of age. The cause of the hypertension is an increased peripheral resistance, the reason for which is not yet understood. Pathologically, its effects show first upon the left ventricle of the heart muscle and, at a later date, there may be some increase in the right ventricle musculature also. Arterio-sclerosis of the main vessels is usually present in the later stages, often affecting the coronary or cerebral arteries, leading to changes in either the heart or brain. In the kidney, changes secondary to the thickening of the arterioles may occur, giving rise to the small red kidney.

Of the symptoms, epistaxis is probably the only one referable directly to the hypertension. Many of the patients feel quite fit and well for some years. Later, complaints of a variety of symptoms, often of very gradual onset, appear. These symptoms include lack of energy, fatigue, restiveness, headaches and giddiness, especially on stooping or severe exertion. Headache is frequent, and is usually worse after exercise or on waking. In women, emotional upsets with nervousness and irritability of temper is a common occurrence, especially after the menopause; loss of the power of concentration and a failing memory for recent events is another common manifestation of essential hypertension. Minor cerebral attacks often occur, with dizziness, faintness or transient loss of consciousness.

The most common cardiac symptom pointing to this disease is palpitation, which may occur either with a normal rhythm, or with extrasystoles, or, in the more advanced cases, with auricular fibrillation. Dyspnoea on exertion is another symptom, which is followed, as the left ventricle begins to fail, by a back pressure effect upon the pulmonary system, leading to the distressing symptom of nocturnal dyspnoea or cardiac asthma. Pain of an anginal type is common, especially in the more advanced cases.

On examination, the patient is usually noticed to be the obese, well-built type, usually of normal colour, except for some slight increase in skin pallor. The apex beat of the heart is usually found to be displaced downwards and to the left, outside the mid-clavicular line. The impulse is heaving and sustained, and, on auscultation, the most significant finding is that the aortic second sound is slapping or ringing in character. Other associated conditions, such as the presence of extrasystolic interruptions or auricular fibrillation may be present coincidentally, and degenerative changes may have taken place, especially in the valves, leading to the appearance of systolic murmurs to colour the picture even further. The blood pressure readings may be found to be in the region of $200-240 \mathrm{~mm}$. $\mathrm{Hg}$. systolic, and I00-IIo mm. $\mathrm{Hg}$. diastolic, or higher. If the arteries of the retina are examined by means of an opthalmoscope, their appearance is, 
characteristically, that of silver wires, i.e., they are tonically contracted and reflect the light directly to the eye.

Essential hypertension has been present usually for some years before a patient seeks advice on account of symptoms, and, therefore, it is questionable whether the finding of a heightened blood pressure at a routine examination, after a period of relaxation, in the absence of symptoms, should be communicated to the patient. Hypertension looms up before the layman as a hideous dreaded disease which may snatch him away in the prime of his life. There is, however, one type in which the course is very much more rapid, and to this, the name of malignant hypertension has been applied. In this, symptoms occur early in the disease and usually at an earlier age. Coronary or cerebral thrombosis or rupture are frequent accompaniments. Furthermore, such patients are liable to cerebral hypertensive attacks, in which, due to a sudden vasoconstriction, the blood pressure rises rapidly to abnormal heights. When this occurs, the patient may have a convulsion and become unconscious, accompanied by cyanosis and stertorous breathing. There may be a transient hemi-or mono-plegia, and aphasia is commonly associated.

At a later date, in hypertension of either type, certain manifestations of renal dysfunction appear. This is shown by an increased output of urine with a low specific gravity of between IOI 5 and IoIo. In many cases, as much urine is passed during the night as during the day, and on examination of the urine microscopically, casts of either the hyaline or granuiar variety may be found. In the later stages, especially in the malignant type, uraemia may be the final chapter in this story. During the last stages of the disease, dyspnoea is the most conspicuous clinical feature, the patient being unable to lie down with any degree of comfort. Even later, CheyneStokes breathing may occur and the patient may sink into an apathetic condition from which there is no recovery. During these later stages, pulsus alternans is often present, indicating failure of the left ventricle; its appearance augurs ill for the patient.

In hypertension due to chronic nephritis, there is usually a history of a preceding attack of acute nephritis, often many vears previously, and the patient may have passed through the second stage of nephritis with the presence of oedema. The differentiation of this type of hypertension from essential hypertension may be difficult clinically, but the following points may be of use: the history of acute nephritis; the age-chronic nephritis is rare after 40 years of age the systolic blood pressure is rarely as high as in the essential type, although the pressure is most stable; an urinalysis will show a specific gravity often below IoIo, marked albuminuria, and, on microscopy, hyaline, granular and red blood-cell casts will be found; an opthalmoscopic examination may reveal the presence of an albuminuric retinitis, with haemorrhages and exudates situated around the mascula of the retina; furthermore, the renal efficiency tests are much more markedly impaired, and eventually symptoms of uraemia will appear.

The cause of death in hypertensive disease is usually heart failure or vascular accidents affecting the cerebral or coronary vessels; more rarely, the patient dies of uraemia.

Hypertension due to chronic lead poisoning is now rather a rarity. The patient, usually a painter by trade, complains of lassitude and symptoms referable to a secondary anaemia. There may be attacks of severe small-intestine colic, with pain situated around the umbilicus and lower abdomen, and, upon examination, a blue line may be found just below the gum edge, and an examination of the blood will show the characteristic basophil strippling.

Increased intracranial pressure is usually due to haemorrhage, tumour, or inflammation, and therefore the high blood pressure will be found as a coincidence in such diseases. Their diagnosis should therefore present no difficulty, because of the associated signs.

The rare suprarenal tumour is a pheochromocytoma arising from the cells of the medulla of the suprarenal gland. In this condition, the hypertension tends to be present in paroxysms, readily induced by exercise or emotion. The diagnosis is important since it is often amenable to surgery with complete relief of symptoms.

Tumours of the cortical cells of the suprarenal produce, in adults, a form of basophilism, known as the adreno-genital syndrome. It is distinguished from the clinical condition produced by a basophil adenoma of the pituitary (Harvey Cushing's syndrome) by the presence of a tumour in the loin (occasionally) by radiology and by the biological assay of the urinary androgens.

The basophilic syndrome includes adiposity, of rapid onset and of "Buffalo" type, kyphosis, sexual dystrophy, hirsutes, especially of face and trunk, the presence of lineca atrophica and a dusky, cyanotic tinge to the skin and marked hypertension.

Obstruction to the free flow of urine by enlargement of the prostate is a cause which must not 
be forgotten, and it has been emphasised how valuable an index is the blood pressure to the surgeon, in his handling of his prostatic cases.

Coarctation of the aorta has recently been brought to the forefront because of the discovery of so many cases in late years. Due to a congenital obstruction to the aorta the circulation to the abdomen and legs has to be maintained by new collateral channels, through the internal mammary, transcervical and other arteries around the scapula. In order to maintain this abnormal circulation, the cardiac output is markedly increased and the blood pressure in the arms is found to be very high, whilst in the legs, the blood pressure is low due to the inefficient circulation. Evidence of the collateral circulation will be seen by the wide, tortuous pulsating arteries around the scapula and in the epigastric arteries. A radiograph of the chest will show how the internal mammary arteries have taken their part in this collateral circulation, their tortuosity notching the ribs (Roesler's sign).

Such, then, is a brief description of some of the more common causes of hypertension. But the other groups given in the classification must be remembered. How far further research will implicate the endocrine glands remains to be seen, but there can be no doubt about the importance of the pituitary gland as an aetiological factor.

We will now turn our attention to the diagnosis of valvular lesions. Aetiologically the most common cause of such valvular lesions is a previous rheumatic infection, which produces changes within the valves of the heart by reason of the fibrous reaction which occurs following such inflammatory changes. Next on the list comes degenerative change, occurring in the more el erly patients, possibly associated with a long-continued strain affecting one of the cardiac valves. Such changes are due to the deposition of calcium in and around a valve which has been damaged through deficient blood supply by occlusion of the vasa vasorum, and a replacement fibrosis follows degeneration of the more delicate coverings of the valve. Congenital malformation arising because the normal development of the heart, in part or whole, has either been arrested or disturbed in some way, is the other cause of valvular disease to be remembered.

The most common valvular lesion is that associated with a rheumatic mitral stenosis. The patient, usually between the ages of 20 and 30 years, complains of increasing dyspnoea on exertion. As already pointed out, it is probable that in the early stages this is due to a deficient pump action of the auriculo-ventricular valves, but at a later stage the dyspnoea is due to cardiac failure, following the supervention of an abnormal cardiac rhythm, usually auricular fibrillation. In the more advanced cases the typical mitral facies will be present, with cyanosis of the lips, tips of the ears and extremities, and the cyanotic flush over the malar region. The pulse, providing no abnormal rhythm has appeared, is usually of regular rate, with a rather lessened amplitude than normal, but the blood pressure readings may be within normal limits. On examination of the heart some enlargement may be found, with the apex beat displaced somewhat to the left. On palpation this enlargement will be verified and a thrill may be present. Classically, this thrill is pre-systolic in time, but it may be either mid-diastolic or systolic. On auscultation, it is usual to find both heart sounds present over the whole of the precordia, but the mitral first sound is usually accentuated and louder than normal. Certain murmurs appear, and are of diagnostic importance. The murmur upon which one bases a clinical diagnosis of mitral stenosis is the mid-diastolic murmur which is usually heard only over a small area at or around the cardiac apex, with little or no propagation. As the stenosis increases, the diastolic murmur may become crescendo in type, leading to the typical pre-systofic murmur which has been described for so many years as the classical sign of mitral stenosis. But it should be emphasised that it is the presence of the mid-diastolic murmur on which one should base the diagnosis of mitral stenosis. This murmur is produced by the flow of blood which occurs from auricle to ventricle in the stage of diastasis before auricular contraction begins. As the stenosis becomes more marked, the auricular contraction forces the blood which has remained in the auricle through the small stenotic valve, and so produces the pre-systolic crescendo murmur. This also explains why this crescendo murmur disappears with the onset of fibrillation. In the earlier cases, however, these two murmurs may not be present, and in its stead one will find an accentuated first sound, followed by a harsh systolic murmur. Diagnosis may then be difficult on clinical grounds, but this systolic murmur is a sign of early stenosis and not a true mitral incompetence. The diagnosis will be confirmed only upon finding the characteristic radioscopic outline of the heart.

Pure mitral incompetence is probably one of the most rare of all valvularlesions, and it is accompanied by the largest heart of all the valvular lesions. The diagnosis can only be made 
by radioscopic examination showing enlargement of both ventricles and the left auricle, after the finding of a harsh systolic murmur which is propagated outwards towards the axila or round to the back. It is emphasised again that usually such a murmur is indicative of early stenosis and not incompetence.

Lesions of the aortic valve are due either to a previous rheumatic infection, to syphilitic disease of the aorta, or to the imposition of a bacterial endocarditis upon a valve already damaged by a previous rheumatic infection, or the site of a congenital defect. Pure aortic stenosis is rare, and in order to make a diagnosis, the following characteristics must be present:-Firstly, the heart must be enlarged, due to hypertrophy of the left ventricle, downwards and to the left, often being found in the 6th space outside the midclavicular line; secondly, a systolic thrill must be felt over the aortic area, that is, the 2nd right costal cartilage area. It may be extremely difficult to feel this thrill with the patient either standing up or lying down. It is usually most marked if the patient leans forward, takes a long breath followed by a full expiration and holds his breath in this position for a few seconds. Thirdly, the cardiac sounds are heard over the whole precordia, often with marked accentuation of the first sound. Over the aortic area, a harsh systolic murmur is heard which is conducted upwards into the root of the neck and can often be heard over the carotid artery. Both aortic first and second sounds are present in pure stenosis, although the systolic murmur may run into the second sound so that it is difficult to hear as a separate entity. Fourthly, the radial pulse has certain characteristics. These include a slow rise, often with an anacrotic wave, definitely palpable, maintenance of pressure and a slow decline-a classical pulsus tardus. The blood pressure readings are usually about I40 systolic and Ioo diastolic-in other words, the pulse pressure is markedly reduced.

More commonly, however, stenosis is associated with some degree of incompetence, due to the fact that the anatomy of the semi-lunar valve has been so altered that it is impossible for the normal apposition of the valve cusps to occur. In this case certain alterations occur. The aortic second sound may be much diminished or even absent, and following upon it one will hear a soft diastolic murmur, or whiff. It is usual to find that this murmur is heard best, not at the aortic area, but either to one side or in the middle of the sternum, 2 to 3 inches below the aortic area. It is produced by, the backward flow of blood from the aorta not being arrested at the semi-lunar valve. This column of blood causes dilatation and hypertrophy of the left ventricle. It is this hypertrophy which accounts for the enormous enlargement of the heart, and it also has one other effect-due to the enlargement and dilatation of the ventricle the auriculo-ventricular valve is pulled upon, causing a degree of relative stenosis. Furthermore, the downward column of blood may actually impinge upon the elongated auriculo-ventricular valve. It is not surprising, therefore, that in the 'stenotic-incompetent' aortic valve there is frequently associated a rough diastolic murmur at the cardiac apex. This is known as the Austin Flint murmur; its presence must be remembered, because it is excessively rare for a syphilitic aortitis to be present at the same time as a rheumatic mitral lesion. This backward flow of blood also produces certain characteristic alterations upon the pulse. As can be imagined the diastolic pressure is considerably lower than normal. It may, in fact, be entirely absent if the incompetence is of very gross degree. Because of this fall in the diastolic fressure, the pulse pressure is high. This will be appreciated on palpating the pulse, especially if the arm be held up towards the level of the head. Since there is no diastolic resistance the pulse is collapsing in type (Corrigan's water-hammer pulse). This excessive pulse pressure will, in the very gross cases, be shown upon the capillary bed if some slight pressure be made upon the nails. But it must be emphasised that the diagnosis of aortic incompetence is made by the finding of the aortic diastolic murmur, and not upon the pulse and capillary characteristics since these only appear in the very late stages, if at all.

An early degree of aortic incompetence may be very difficult to diagnose, since none of the signs are present. The only auscultatory sign is a soft, diminuendo whiff, discovered on careful auscultation down one or other side of the sternum and not the aortic area.

The pulmonary valve is rarely the seat of primary disease alone, but congenital lesions of the valves most commonly occur at this valve. Relative stenosis caused by increased back pressure in the pulmonary circuit is a fairly common occurrence, especially in the late stages of mitral stenosis. In the more severe grades of pulmonary stenosis of congenital origin, there are usually other associated malformations of the heart, and of these the tetralogy of Fallot is described. In this syndrome there is a defective interventricular septum, pulmonary stenosis, hypertrophy of the right ventricle and dextraposition of the aorta. The patency of the interventricular 
eptum is to some extent the compensatory mechanism, but it may, if large enough, involve the whole auriculo-ventricular bundle and so produce congenital heart block also. Pulmonary stenosis is diagnosed by the finding of a systolic thrill and murmur over the pulmonary area, with diminution or absence of the pulmonary second sound. The patient, especially in the more severe degrees, is cyanosed, and clubbing of the fingers is a common accompaniment. Relative stenosis occurs in cases of advanced mitral stenosis and is the cause of the systolic murmur which is heard in that area in this condition, first described by Grahame Steele and bearing his name.

The diagnosis of lesions of the tricuspid valve is more difficult, but lately stress has been laid upon this clinical condition by the accurate observation of some cases. The clinical characteristics of tricuspid stenosis are as follows:-

It usually occurs in women of between 40 and 50 years of age, who give a history of previous rheumatic carditis. They complain of dyspnoea and fullness and swelling of the abdomen, and upon examination marked ascites with enlargement of the liver out of all proportion to the degree of cyanosis and systematic venous circulation is present. Marked systolic pulsation of the jugular veins is often a prominent feature. In some cases there may be an associated jaundice, often paroxysmal in occurence. The liver will also be found to exhibit pre-systolic pulsation, and a pre-systolic murmur will be heard at the lower end of the sternum, about the epigastric region; confirmation of these findings will be given if a barium swallow is watched on the radioscopic screen, when deviation of the oesaphagus to the left will be a prominent feature.

Tricuspid incompetence is often much more difficult to diagnose, but it is often present in the later stages of heart failure, and in this condition a distinct systolic murmur is usually heard over the tricuspid area, quite limited in distribution.

It must not be thought that each valve is implicated singly in any disease process-quite the contrary, since in many of the cases there are several valves implicated together. The diagnosis, however, of any of these lesions should be effected if the points already mentioned are borne in mind.

The differential diagnosis of a systolic murmur heard over the precordia often presents many difficulties, although some of the causes have already been mentioned. The pigeon-holing of such a systolic murmur into the group of functional or haemic murmurs used to be the delight of many clinicians but it is becoming increasingly obvious that functional or haemic murmurs are of rare occurrence. This is well illustrated by the following case:

A young girl of $\mathbf{I} 4$ years, when examined by her school doctor at a routine examination, was discovered to have a systolic murmur over the mitral area. She apparently had no symptoms at all, and had led a normal existence until this finding. On careful examination of the heart no physical signs other than this systolic murmur over the mitral area could be found, but on taking the blood pressure one was disconcerted to find that it was $190 /$ roo. The arteries of the lower limb were therefore palpated, and it proved impossible to find either the dorsalis pedis, the posterior tibial or the popliteal arteries, and the femoral artery was only slightly pulsatile. The diagnosis of coarctation of the aorta was therefore made, and this was confirmed by the finding of Roesler's sign upon the ribs in a radiograph of the chest. Careful enquiry of the mother then elicited the information that the child had had several attacks of epistaxis, for which no adequate explanation had been given at the time.

Congenital lesions of the heart are responsible for many of the murmurs heard. In the more severe grades they are usually associated with a systolic thrill, in fact it has been said that without the finding of a systolic thrill, diagnosis is well-nigh impossible. Patency of the interventricular septum is usually associated with a systolic thrill and murmur localised to the third left intercostal space. This was first described by Roger, and is therefore known as the "maladie de Roger." It is not usually associated with any other signs or symptoms, and the patient usually lives to near the normal span. Patency of the interauricular septum causing a systolic murmur is more difficult to diagnose without radioscopic screening, when it will be found that the pulmonary artery and hilar shadows are markedly pulsatile (dance de hilar). A systolic murmur is often present over some part of the precordia, usually in the midsternal legion. A patent ductus arteriosus may be variable in the symptoms and signs it produces but usually it can be diagnosed by the finding of both a systolic and diastolic murmur over the first left intercostal space, or second left rib. It is usually rough and harsh in character and is given the name of "the machinery" or "cog-wheel" murmur. In the more severe cases it may produce such a reflux of blood from the aorta to the pulmonary artery that all the signs associated with an advanced degree of aortic incompetence are present as well; but the murmur is characteristically distinctive. Congenital pulmonary stenosis has already been mentioned. A congenital bicuspid 
valve in the aortic area may produce a systolic murmur in the aortic area. The diagnosis made difficult unless there is an associated stenosis producing a thrill and the usual pulse features. It is important because it is sometimes associated with the superposition of subacute bacterial endocarditis. The systolic murmurs described by Austin Flint and Grahame Steele have already been mentioned, and it is important to $r \in m \in m b e r$ that they occur very frequently. The systolic murmur in the mitral area, appearing for the first time in late adult life, may be the first sign of aortic reflux from syphilitic aortitis, and it will require very careful auscultation to hear the early diastolic whiff in the midsternal region. In children especially, severe exercise or increased cardiac output from any cause, but particularly that associated with fevers, may produce a soft, blowing systolic murmur at the mitral area, and this finding often leads to unnecessary worry by both the doctor and the patient's parents. It is only discovered whilst the cardiac rate is accelerated, and is very soft and blowing in nature. The resumption of the normal cardiac rhythm is soon followed by the disappearance of this murmur. In pericarditis, especially when of slight degree, a soft murmur, systolic in time, may be found over some part of the precordia, but careful auscultation will disclose its nature in most cases, and it may be followed by a very short diastolic murmur of similar character, and these sounds may be intensified by pressure with the stethoscope. Characteristically, the sounds can be described by the words "frou-frou." Exocardial murmurs are often systolic in time, as are the cardio-respiratory murmurs. Many of them can be made to disappear by instructing the patient to hold his breath, either in the position of maximum inspiration or maximum expiration.

A haemic murmur associated with a severe anaemic may appear at any part of the precordia. They are soft murmurs, which are markedly influenced by the position of the patient, often being audible only when the patient is lying down, or being intensified in that position. These murmurs are always systolic in time, often not starting until half-way through systole and always ending before the second sound appears. But a haemic murmur must never be diagnosed unless there is evidence of a severe anaemia.

From consideration of these points it will be seen that it is usually possible to make the $\sigma$ diagnosis of the cause of a systolic murmur much more concrete than was at one time considered $\delta$ possible by clinical methods alone. One has not attempted to cover the whole range of possibles diagnosis which might be attempted by cardiologists, but it is hoped that such points as have been made will help to stimulate more exact diagnosis of the causes of systolic murmurs and to $\$$ place them in their correct perspective in relation to prognosis.

A. RAE GILCHRIST, Edinburgh Post-Graduate Lectures, 1940-4 I, 2, 70.

\title{
Clinical Page
}

\section{THREE CASES ILLUSTRATING RARE DISEASES OF THE ILEO-CAECAL REGION}

\author{
By A. E. MORTIMER WOOLF, M.B., B.Ch.(CAntaB), F.R.C.S. \\ (Senior Surgeon Queen Mary's Hospital for East End; Surgeon to the E.M.S., etc.).
}

Ileo-caecal tuberculosis is a rare disease. Of recent years its incidence has diminished, probably because many cases in the past have been wrongly classified. Since Crohn described the condition of terminal ileitis, it has become apparent that this condition (the causal agent of which is quite unknown) embraced many cases erroneously thought to be tuberculosis. The following experience is an example of this error.

In I924, I was consulted by a man of $3 I$ with a lump in the right iliac fossa. This had been diagnosed as appendicitis and an operation had been performed at a London hospital. The man was having indigestion, pain, probably of an obstructive character, and was losing weight. He looked ill, and there was a small healed scar about one inch long just inside the antero-superior spine. A mass could be felt in the right iliac fossa. I diagnosed ileo-caecal tuberculosis. At the time I was unable to prove or disprove this, because the patient made me promise that if he consented to operation, I was to remove no part of his anatomy. $\mathrm{He}$ 